\title{
Article \\ Brain Symmetry Analysis during the Use of a BCI Based on Motor Imagery for the Control of a Lower-Limb Exoskeleton
}

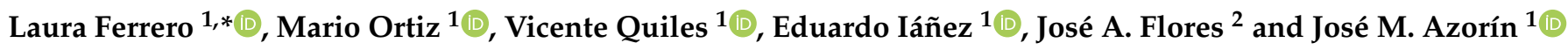 \\ 1 Brain-Machine Interface System Lab, Miguel Hernández University of Elche, 03202 Elche, Spain; \\ mortiz@umh.es (M.O.); vquiles@umh.es (V.Q.), eianez@umh.es (E.I.); jm.azorin@umh.es (J.M.A.) \\ 2 Department of Engineering, Miguel Hernández University of Elche, 03202 Elche, Spain; ja.flores@umh.es \\ * Correspondence: lferrero@umh.es
}

Citation: Ferrero, L.; Ortiz, M.; Quiles, V.; Iáñez, E.; Flores, J.A.; Azorín, J.M. Brain Symmetry Analysis during the Use of a BCI Based on Motor Imagery for the Control of a Lower-Limb Exoskeleton. Symmetry 2021, 13, 1746. https:// doi.org/10.3390/sym13091746

Academic Editor: Fabrizio Vecchio

Received: 30 July 2021

Accepted: 13 September 2021

Published: 19 September 2021

Publisher's Note: MDPI stays neutral with regard to jurisdictional claims in published maps and institutional affiliations.

Copyright: (c) 2021 by the authors. Licensee MDPI, Basel, Switzerland. This article is an open access article distributed under the terms and conditions of the Creative Commons Attribution (CC BY) license (https:// creativecommons.org/licenses/by/ $4.0 /)$.
Abstract: Brain-Computer Interfaces (BCI) are systems that allow external devices to be controlled by means of brain activity. There are different such technologies, and electroencephalography (EEG) is an example. One of the most common EEG control methods is based on detecting changes in sensorimotor rhythms (SMRs) during motor imagery (MI). The aim of this study was to assess the laterality of cortical function when performing MI of the lower limb. Brain signals from five subjects were analyzed in two conditions, during exoskeleton-assisted gait and while static. Three different EEG electrode configurations were evaluated: covering both hemispheres, covering the non-dominant hemisphere and covering the dominant hemisphere. In addition, the evolution of performance and laterality with practice was assessed. Although sightly superior results were achieved with information from all electrodes, differences between electrode configurations were not statistically significant. Regarding the evolution during the experimental sessions, the performance of the $\mathrm{BCI}$ generally evolved positively the higher the experience was.

Keywords: brain symmetry; Brain-Computer Interfaces; electroencephalography; exoskeleton; motor imagery; laterality; hemisphere specialization

\section{Introduction}

Motor imagery (MI) is defined as the process of imagining or performing a mental execution of a movement without any actual muscle activation. MI, the observation of an action and the actual motor execution produce similar brain patterns [1]. There are different techniques to measure brain activity, with electroencephalography (EEG) being one of the most commonly used [2].

Sensorimotor rhythms (SMR) are brain oscillations recorded over the sensorimotor cortex. Motor processing produces changes in the ongoing SMR as event-related desynchronization (ERD) and synchronization (ERS). The phenomenon of ERD in alpha and beta band rhythms is associated with cortical areas that are ready to prepare a movement, and ERS is correlated with resting areas [3].

MI has been frequently employed in Brain-Computer Interfaces (BCI) [4]. BCIs are systems that record brain activity, for instance with EEG, and decode this information to generate commands for controlling external devices. In the case of MI, BCI must identify this brain pattern from the EEG.

Different devices have been commanded by means of $\mathrm{BCI}$, including robotic orthosis and exoskeletons that can provide motor assistance for people with motor disabilities $[5,6]$.

There are different $\mathrm{BCI}$ control paradigms for this kind of devices. The most commonly used are based on external stimuli such as steady-state visually evoked potentials [7] or auditory evoked potentials [8], and those related with real motion or MI such as motionrelated cortical potentials [9-11] or ERD/ERS [12-16]. Controlling a BCI with MI can emulate the lost motor function by inducing mechanisms of neuroplasticity [17]. 
The brain's anatomical structure and function varies between left and right hemispheres. There are neural functions and cognitive processes that tend to be specialized to one hemisphere or the other, and this property is defined as brain laterality [18]. Some of them include face and body perception and motor skills [19]. Each brain hemisphere controls the contralateral side of the body, and the natural asymmetry of the brain is the cause of left/right side dominance, i.e., better performance or preference for using one hand, foot or eye [20,21]. Different models of lateralization have been proposed: homogeneous lateralization, which considers the dominance of all organs from the same side; crossed lateralization, which contemplates different dominances; and the undefined model, which has the hypothesis that the dominance cannot be determined [20].

Virtual reality has been employed to study lateralization among individuals, revealing that the performance of real motion tasks with the dominant hand is similar to the one obtained with both hands. In addition, this trend is also noticeable with the dominant body side for controlling the posture to perform certain tasks [20].

Previous studies have attempted to discern between right hand and left hand MI [12]. The effects of handedness on the SMR desynchronization have also been assessed. Zapala et al. [22] compared the performance of an upper-limb MI based BCI between left- and right-handed individuals. This BCI employed common spatial patterns (CSP) to extract features that could be used to distinguish between left and right MI classes, concluding that left-handed participants showed lower accuracy. Additionally, the power of the signal during MI was studied in different brain regions. Left-handed participants showed no differences in SMR in the right parietal brain region between right and left hand MI. However, right-handers showed higher lateralization. Shieh et al. [23] designed an algorithm that identified the best spectral and spatial patterns to discriminate between left and right hand MI. Results showed that for left MI, spatial patterns covered right hemisphere areas and the opposite way for right MI.

Regarding lower-limb, since the foot brain area is located in the interhemispheric fissure, left and right lower-limb areas are close. Therefore, it is difficult to distinguish between each limb movement or MI, since they produce similar EEG patterns. Pfurtscheller et al. [24] showed that hand MI produces a mu band (around $10 \mathrm{~Hz}$ ) ERD, while foot MI produces an ERS in the hand cortical representation area with no laterality influence. However, a clear subject dependency was also reported. Tariq et al. [25] analyzed EEG patterns when performing right and left foot dorsiflexion MI and designed a BCI that had to discriminate between both types of MI. In mu frequency band, ERD/ERS patterns showed a lateralized distribution during left and right foot MI. Regarding beta frequency band, ERD/ERS showed a power concentration at the vertex, which is the cortical foot area representation [25]. In addition, contralateral dominance was found during right foot MI in electrode C3 for ERS patterns [26].

Crémers et al. [27] and Jahn et al. [28] observed right hemisphere specialization during MI of the gait. This brain task was found to be associated with activity in frontal and parietal areas, mainly on the right hemisphere and on the left side of the cerebellum [27].

The aim of this paper is to evaluate the performance of a BCI to control a lowerlimb exoskeleton when using information from dominant, non-dominant and both brain hemispheres. We thereby assess whether there is a hemisphere specialization during MI of gait. In addition, since subjects participated in several sessions, this research studies the evolution of performance with practice as sessions go on, as well as whether and how this hemisphere specialization is affected.

\section{Materials and Methods}

\subsection{Subjects}

Five healthy subjects participated in the study (mean \pm age, $23.2 \pm 1.3$ ). They did not report any known disease and had no movement impairment. They did not have any previous experience with BCI. All participants were right-handed and right-footed, with four of them being men and one female. They were informed about the experiments 
and signed an informed consent form in accordance with the Declaration of Helsinki. All procedures were approved by the Responsible Research Office of Miguel Hernández University of Elche (Spain).

\subsection{Equipment}

Electroencephalography (EEG) was employed to record brain activity with a set of 32 slim electrodes (Brain Products GmbH, Germany) positioned on a 64-electrode actiCap (Brain Products $\mathrm{GmbH}$, Germany). Four electrodes were located next to the eyes for recording electrooculography (EOG). Ground and reference electrodes were located on the right and left ear lobes, respectively. The remaining 27 electrodes were placed over the cap following the 10-10 international system: F3, FZ, FC1, FCZ, C1, CZ, CP1, CPZ, FC5, FC3, C5, C3, CP5, CP3, P3, PZ, F4, FC2, FC4, FC6, C2, C4, CP2, CP4, C6, CP6, P4. Signals were amplified with an actiCHamp module (Brain Products $\mathrm{GmbH}$, Germany) and transmitted wirelessly with MOVE (Brain Products $\mathrm{GmbH}$, Germany) to pyCorder recorder software (Brain Products GmbH, Germany).

Subjects wore a H3 exoskeleton (Technaid, Madrid, Spain), which provides assistance for walking. The subjects used crutches for extra support and a member of the research staff was behind them to prevent any possible risk of falling in case of loss of balance. Control commands and exo status were sent and received via Bluetooth. Figure 1 shows the experimental setup.
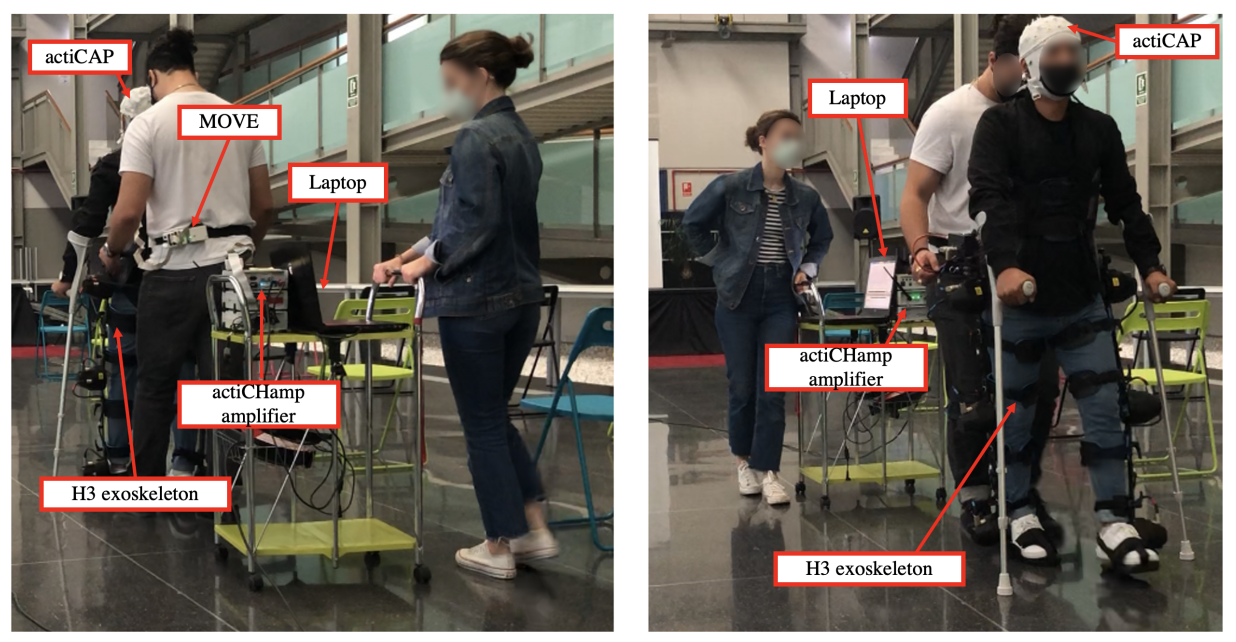

Figure 1. Experimental setup. Two operators were present during each session. One of them carried the equipment and the other one was behind each participant for safety reasons.

\subsection{Experimental Design}

The experimental design was the same presented in [16]. Each subject performed 5 sessions, which were divided in training and testing. During training, the exoskeleton was in opened-loop control, which means it was controlled with predefined commands sent by the laptop. Then, during the test phase, the exoskeleton was controlled by commands issued in real time from the BCI based on the EEG patterns of the subject. Only the training data were employed for this research. Figure 2 shows the schema of the protocol.

For training, each subject performed 22 trials. They had to perform a sequence of three mental tasks: MI of the gait, idle state, and a regressive count. For idle state, participants were asked to be as relaxed as possible. As in this research the attention mental task is not analyzed, only data from MI and idle states were considered.

During the use of an exoskeleton, there are two possible states that the subject can be in: standing and moving. Due to that, MI must be analyzed in both states which makes necessary the creation of two different classifier models. One is based on the traditional definition of MI, while the other one combines MI with motor execution. This way, half 
of the 22 trials were performed in a full standing status (full-static) with the exoskeleton without performing any movement, 11 trials, and the other half were performed in a full motion status (full-motion) assisted by the exoskeleton, 11 trials. For both conditions, the sequence of mental tasks was identical.

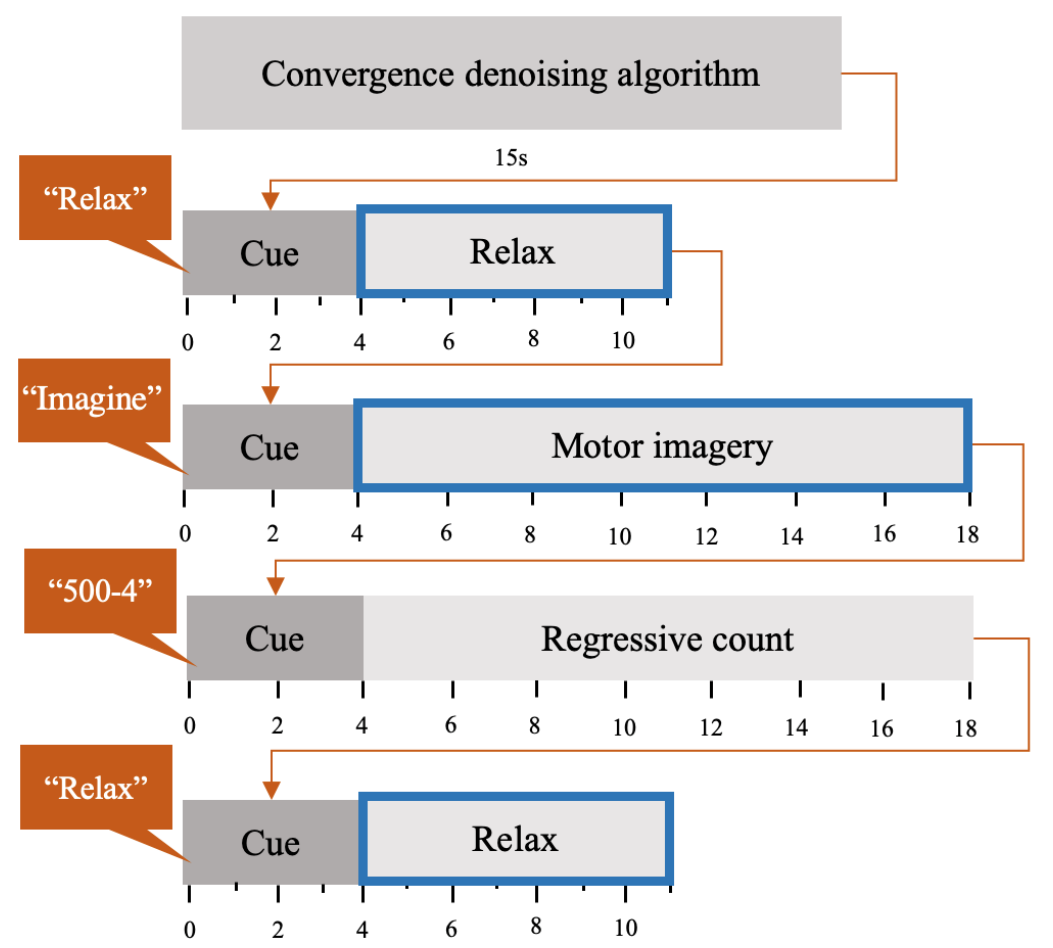

Figure 2. Schema of the protocol for each trial. This sequence was repeated 11 times while static and 11 with exoskeleton-assisted gait. Only information from events with blue border was considered for this research.

\section{4. $B C I$}

\subsubsection{Acquisition}

EEG signals were acquired at $200 \mathrm{~Hz}$ sample ratio. All the analysis were done following a pseudo online approach to simulate a real-time application. Epochs of $1.5 \mathrm{~s}$ were processed with $0.5 \mathrm{~s}$ of shifting. Therefore, the processing of each epoch must be performed in less than $0.5 \mathrm{~s}$.

\subsubsection{Pre-Processing}

EEG signals went through two hardware filters assigned in the pyCorder app (BrainProducts, Germany): a Notch filter at $50 \mathrm{~Hz}$ to remove the contribution of the power line and a high-pass filter at $0.5 \mathrm{~Hz}$. Afterwards, $H_{\infty}$ denoising framework was applied [11]. This algorithm employs EOG signal, estimates its contribution to each EEG channel and removes it. In addition, it also estimates and removes any signal drift.

\subsubsection{Processing}

Three different methodologies were employed for feature extraction: Filter Bank Common Spatial Patterns (FBCSP) [29], Stockwell Transform (ST) [30], and Welch's Power Spectral Density (PSD) estimation [31].

\section{FBCSP}

FBCSP applies Common Spatial Patterns (CSP) [32] at different frequency ranges. For this research, EEG signals went through 4 band-pass filters that included alpha and beta rhythms: $5-10 \mathrm{~Hz}, 10-15 \mathrm{~Hz}, 15-20 \mathrm{~Hz}$, and $20-25 \mathrm{~Hz}$. CSP transforms EEG data 
to construct optimal spatial filters that aim to discriminate between two different brain patterns. In this case, it must discriminate between MI and idle state.

First, covariance matrices are computed for each class, MI and idle state, and normalized as in

$$
C_{1}=\frac{X_{1} X_{1}^{T}}{\operatorname{trace}\left(X_{1} X_{1}^{T}\right)}, C_{2}=\frac{X_{2} X_{2}^{T}}{\operatorname{trace}\left(X_{2} X_{2}^{T}\right)} .
$$

where $X$ are EEG data from each class that have $N$ number of channels and $T$ number of samples, $N * T$.

These matrices are computed for each trial and then all the matrices from the same class are averaged: $\overline{C_{1}}$ and $\overline{C_{2}}$. Afterwards, $\overline{C_{1}}$ and $\overline{C_{2}}$ are combined into the composite spatial covariance matrix, and it is factorized as

$$
C=\overline{C_{1}}+\overline{C_{2}}=U_{0} \Sigma U_{0}^{T} .
$$

$\Sigma$ is the diagonal matrix of eigenvalues, and $U_{0}$ is a matrix of eigenvectors. The results of the decomposition are combined as

$$
\begin{gathered}
P=\Sigma^{1 / 2} U_{0}^{T}, \\
S_{1}=P \overline{{C_{1}}} P^{T}, S_{2}=P \overline{{C_{2}}_{2}} P^{T} .
\end{gathered}
$$

$S_{1}$ and $S_{2}$ have the same eigenvectors, and the sum of both matrices of eigenvalues is the identity matrix.

$$
S_{1}=U \Sigma_{1} U^{T}, S_{1}=U \Sigma_{2} U^{T} \text { and } \Sigma_{1}+\Sigma_{2}=I .
$$

The matrix of spatial filters is obtained as

$$
W=U^{T} P .
$$

where $Z$ is the projection of the original EEG signal $S$ into another space. Columns of $W^{-1}$ are the spatial patterns.

$$
Z=W X
$$

$Z$ has the same dimensions as the original signal $X$. However, first and last rows are filters that can discriminate better in terms of their variance. Consequently, as final features, only $m$ first and last components of $Z$ are considered, resulting in $Z_{p}$. Finally, the variances of each component are computed and normalized with the logarithm as

$$
f_{p}=\log \frac{\operatorname{var}\left(Z_{p}\right)}{\sum_{i=1}^{2 m} Z_{p}} .
$$

$f_{p}$ is the vector of features with dimension (fbands $\left.* 2 * m\right) * T$. $m$ was set to 4 so the dimension is $32 * T$.

\section{ST and Welch's PSD}

Before applying ST and Welch's estimation, EEG signals were filtered with spatial Laplacian filter [33]. It enhances the local activity of each electrode while reducing spatial noise.

ST performs a time-frequency decomposition [30]. It performs a phase correction of the wavelet transform with a window that is a Gaussian function of the frequency. The output for each channel is the spectrum of the signal represented as the amplitude with respect to the time and frequency. Frequencies considered were alpha and beta rhythms 8-14 and 20-30 Hz, and a summatory was computed with the amplitude of all of them.

Welch's methodology is employed for estimating the power spectral density (PSD). It is implemented by dividing the signal into overlapping segments, each segment is windowed and then the periodogram is computed for each of them. Finally, PSD is calculated by 
averaging the periodogram of all the segments. Following the same procedure as ST, power of frequencies in alpha and beta bands were added.

Only information from 18 electrodes-FC3, FC1, FCZ, FC2, FC4, C3, C1, CZ, C2, C4, CP3, CP1, CPZ, CP2, CP4, P3, PZ, P4-was used for ST and Welch's method. Electrodes covered premotor area, supplementary motor area, primary motor cortex, inferior and superior parietal lobule.

The vector of features had different dimensions based on the processing method: $1 \times 32$ in FBCSP, and $1 \times$ number of electrodes in ST and Welch's estimation.

\subsubsection{Classification}

Once all the features were extracted, a leave-one-out cross-validation was performed for each subject and session. Furthermore, full-motion and full-static trials were evaluated separately by two independent models. The classifier employed was a Linear Discriminant Analysis (LDA) [34]. It has been commonly used on previous investigations $[16,35,36]$ due to its high reported precision and short computational time $[37,38]$. Consequently, all fullstatic trials for each subject and experimental session were considered for cross-validation. For each iteration of the algorithm, all trials but one were employed for training the model, testing it with the remaining one. The same process was done with full-motion trials.

\subsubsection{Evaluation}

For the evaluation, the percentage of epochs correctly classified was calculated. Then, this value was averaged thorough all the steps of cross-validation.

\section{Results}

All the subjects performed five sessions. However, the data considered for analysis of S4 included four sessions, as the first one was discarded due to the presence of glitches in the signal due to wireless communication problems.

Although all subjects performed eleven trials per session in full-motion and eleven in full-static, some of them were not considered for the analysis. Performing MI while wearing an exoskeleton is a complex task, so it is easy for users to get distracted [16]. Therefore, those trials statistically considered as outliers were removed. Consequently, $7.54 \pm 1.77$ trials in full-static and $7.33 \pm 1.86$ trials in full-motion were selected from each subject.

\subsection{Methodology Influence}

As indicated in Section 2.4.3 several methodologies were used for extracting the MI brain patterns. Table 1 shows the average accuracy obtained with them: FBCSP, ST and Welch's method, during the full-motion and full-static trials.

Table 1. Performance of different methodologies over all sessions: during motor imagery (MI) events (\%MI), during idle state (\%Relax) and total (\%Total). With three different methodologies: Filter Bank Common Spatial Patterns (FBCSP), Stockwell Transform (ST) and Welch's Power Spectral Density estimation (Welch'sPSD).

\begin{tabular}{ccccc}
\hline & & FBCSP & ST & Welch's PSD \\
\hline \multirow{3}{*}{ Full-static } & \%MI & $64.7 \pm 6.5$ & $58.0 \pm 6.4$ & $62.6 \pm 7.6$ \\
& \% Relax & $67.5 \pm 8.9$ & $62.5 \pm 8.0$ & $65.1 \pm 6.1$ \\
& \%Total & $66.1 \pm 6.6$ & $60.2 \pm 5.1$ & $63.8 \pm 4.6$ \\
\hline \multirow{3}{*}{ Full-motion } & \%MI & $61.0 \pm 8.5$ & $56.8 \pm 8.6$ & $60.0 \pm 10.0$ \\
& \% Relax & $62.5 \pm 8.0$ & $55.5 \pm 12.4$ & $58.8 \pm 11.0$ \\
& \%Total & $61.7 \pm 6.2$ & $56.1 \pm 7.3$ & $59.4 \pm 8.3$ \\
\hline
\end{tabular}

A statistical analysis was performed to examine the differences among the methodologies in terms of accuracy. Prior to performing one-way repeated-measures ANOVA 
test, some assumptions must be verified. This process was repeated for full-static and full-motion trials.

- Full-static: The accuracies of two trials were detected as outliers (one for Welch's PSD and one for FBCSP). They were removed as well as the same value for all methodologies to be compared. Afterwards, Shapiro-Wilk test was employed to verify if the data from each group followed a normal distribution and check if the normality null hypothesis could not be rejected ( $p$-value $>0.01$ ). In addition, the sphericity assumption was assessed with Mauchly's test. Results from the ANOVA test showed that the performance of FBCSP, ST and Welch's method were statistically different ( $p$-value $<0.01$ ). Afterwards, pair-wise $t$-test comparisons were conducted, detecting that all methodologies differed significantly from each other $(p$-value $<0.01)$.

- Full-motion: The accuracies of 10 trials were identified as outliers and removed (six for ST and four for FBCSP). Data from each methodology followed a normal distribution ( $p$-value $>0.01)$, and the sphericity assumption was not violated. Results from ANOVA showed significant differences among methodologies $(p$-value $<0.01)$ showing the pair-wise $t$-test comparisons differences between CSP and ST and between ST and Welch PSD ( $p$-value < 0.01).

As CSP showed the highest global accuracy, this approach was the one chosen for the rest of the research. Further analysis will be focused only on this methodology.

\subsection{Subject Influence}

Previous studies have identified differences among subjects in the usage of BCI [39], so subject differences were tested in terms of accuracy. Table 2 shows the average performance for each of the subjects. The differences were analyzed with a one-way ANOVA test. The distribution of outliers that were excluded from the statistical analysis is shown in Supplementary Material, Figure S1.

Table 2. Subject differences in performance during motor imagery (MI) events (\%MI), during idle state (\%Relax) and in total (\%Total).

\begin{tabular}{ccccccc}
\hline & & S1 & S2 & S3 & S4 & S5 \\
\hline \multirow{4}{*}{ Full-static } & \%MI & $59.5 \pm 7.6$ & $70.4 \pm 6.4$ & $63.4 \pm 4.6$ & $64.7 \pm 6.6$ & $65.4 \pm 4.0$ \\
& \% Relax & $69.4 \pm 7.1$ & $67.7 \pm 7.5$ & $63.1 \pm 3.3$ & $69.2 \pm 16.0$ & $67.8 \pm 10.5$ \\
& \%Total & $64.4 \pm 6.2$ & $69.0 \pm 5.7$ & $63.3 \pm 1.7$ & $67.2 \pm 11.2$ & $67.2 \pm 11.2$ \\
\hline \multirow{3}{*}{ Full-motion } & \%MI & $62.8 \pm 7.7$ & $64.3 \pm 9.0$ & $54.9 \pm 9.0$ & $64.2 \pm 11.0$ & $59.3 \pm 5.4$ \\
& \% Relax & $64.5 \pm 3.5$ & $62.7 \pm 6.0$ & $60.7 \pm 11.5$ & $65.1 \pm 13.0$ & $59.8 \pm 6.0$ \\
& \%Total & $63.6 \pm 3.0$ & $63.5 \pm 5.5$ & $57.8 \pm 5.4$ & $57.8 \pm 5.4$ & $57.8 \pm 5.4$ \\
\hline
\end{tabular}

- Full-static: The accuracies of 10 trials were identified as outliers (four for S1, three for S2 and three for S3) and removed. Afterwards, Shapiro-Wilk test was applied, and results showed that the data from each subject followed a normal distribution ( $p$-value $>0.01$ ). Finally, the last assumption to check in order to apply the one-way ANOVA was the data homoscedasticity. This assumption was fulfilled, as assessed by Bartlett test ( $p$-value $>0.01)$. The ANOVA test showed significant differences among subjects ( $p$-value $<0.01$ ), and the pair-wise $t$-test indicated that $S 1$ had a different behavior than the rest $(p$-value $<0.01)$.

- Full-motion: The accuracies of eight trials were detected as outliers (one for S2, one for S3, one for S4 and five for S5) and removed from the analysis. Data from each subject were normally distributed as assessed by Shapiro-Wilk test ( $p$-value $>0.01)$. However, the homoscedasticity assumption was violated, $p$-value $<0.01$ from the Barlett test. Therefore, a non-parametric Kruskal-Wallis test was employed. Results from this test showed no significant differences among subjects ( $p$-value $>0.01$ ). 


\subsection{Training Evolution and Differences between Hemispheres}

As there was a subject dependency, this section analyzes the laterality individually per subject with FBCSP. For the laterality analysis, the performance of FBCSP was assessed using the information from all electrodes, using only the ones located on the dominant hemisphere and using the ones located on the non-dominant hemisphere. Due to the fact that all the subjects were right-footed, the left brain hemisphere was considered as dominant. In this section, the evolution of the performance with the sessions was also assessed, as can be seen in the different images of Figure 3. Furthermore, it was determined whether the best electrode configuration changed with training in terms of performance.
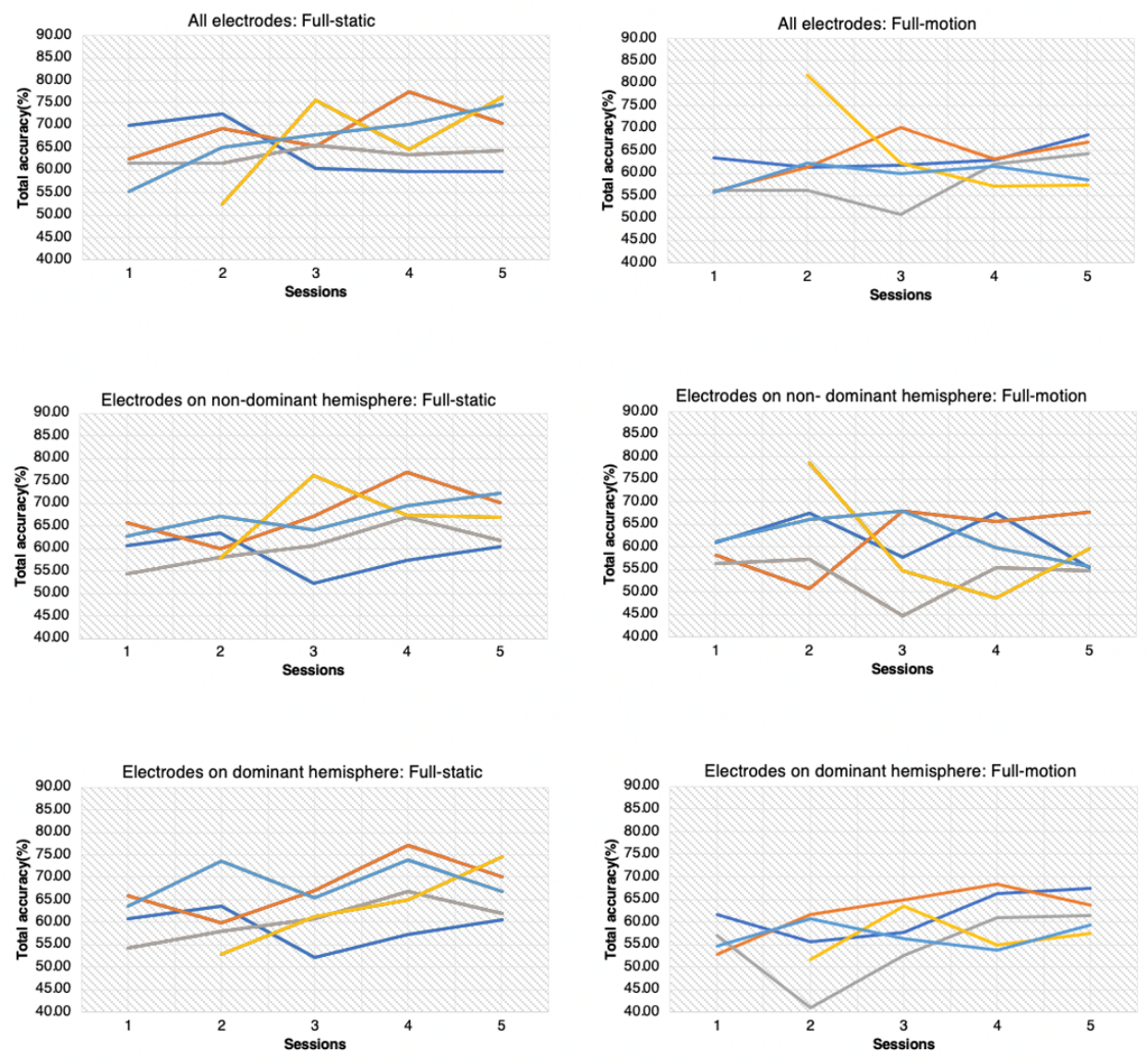

$$
-\mathrm{s} 1-\mathrm{s} 2-\mathrm{s} 3-\mathrm{s} 4-\mathrm{s} 5
$$

Figure 3. Training evolution for full-static and full-motion trials with different electrode configurations: all electrodes, only electrodes located on non-dominant hemisphere and electrodes on dominant hemisphere.

In sum, the statistical analysis of three hypotheses was per subject: differences among sessions, differences among electrode configurations and the interaction between both.

\subsubsection{S1}

- Full-static: Data of all sessions and electrode configurations did not follow a normal distribution. Therefore, instead of performing a two-way ANOVA test, three independent Kruskal-Wallis tests were employed: one assessing differences among sessions, another one with electrode configurations and the third one combining both variables as a new grouping variable. No differences were found for any group ( $p$-value $>0.01$ ).

- Full-motion: Initially, the ANOVA assumption for a normal distribution of the data was not fulfilled. The accuracies of 4 trials were detected as outliers and removed (one for EEG configuration with all electrodes, one for electrodes on the non-dominant 
hemisphere and two for the electrodes on the dominant hemisphere). With the remaining data, all the ANOVA test assumptions were matched, and results did not show statistically relevant differences ( $p$-value $>0.01$ ).

\subsection{2. $\mathrm{S} 2$}

- Full-static: The ANOVA analysis of the results verified that there were not differences among the sessions and the electrode configurations. In addition, the interaction between the sessions and the configurations was not significant.

- Full-motion: ANOVA test revealed significant differences among sessions ( $p$-value $<0.01$ ). Sessions 1 and 2 showed worse accuracy than 3, 4, and 5, as was assessed with a pair-wise $t$-test.

\subsubsection{S3}

- Full-static: ANOVA test did not reveal statistically significant differences among sessions and electrode configurations. Furthermore, the interaction betweeen sessions and electrode configurations was not significant ( $p$-value $>0.01$ ).

- Full-motion: ANOVA test only indicated differences among sessions, and pair-wise $t$-test indicated that the behavior of session 3 was significantly worse in comparison with 1,4 and 5 .

\subsubsection{4}

- Full-static: Data of all sessions and electrode configurations did not follow a normal distribution. Four outliers were detected and discarded (three for the EEG configuration with all electrodes and one for the configuration of electrodes on dominant hemisphere). However, data of all groups still did not follow a normal distribution. Three independent Kruskal-Wallis tests were performed as in S1. Results showed that there were statistically significant differences among sessions ( $p$-value $<0.01)$. Performance of session 2 differed from the one of sessions 3 and 5 , as verified with Dunn's Kruskal-Wallis Multiple Comparisons. In addition, the interaction between electrode configurations and sessions was significant $(p$-value $<0.01)$. This means that the performance of the $\mathrm{BCI}$ with each electrode configuration varied from session to session. The performance of session 2 was better than sessions 3 and 5 with two electrode configurations, all electrodes and electrodes on the non-dominant hemisphere. In the case of the setup on the dominant-hemisphere, the performance of session 2 was worse.

- Full-motion: The performance was statistically different among different sessions, as assessed by ANOVA test. Furthermore, the interaction between session and configuration was statistically significant $(p$-value $<0.01)$.

\subsubsection{S5}

- Full-static: ANOVA test revealed significant differences among sessions ( $p$-value $<0.01$ ), and session 1 was significantly worse than session 5 , as assessed with pair-wise $t$-test.

- Full-motion: Results from ANOVA verified that there were no differences among sessions ( $p$-value $>0.01$ ).

Slightly superior results were achieved with the electrodes covering both hemispheres. However, no statistically significant differences were found among the three electrode configurations. In S4, the evolution of performance with practice was dependent on the selected configuration of electrodes.

On the other hand, the accuracy evolved positively with more training, but the growth was not completely linear. In fact, in some cases, the accuracy dropped after the fourth session. Comparing both types of trials, this positive trend was more evident in the full-static ones. 


\section{Discussion}

\subsection{Study Limitations}

It should be pointed out that the present research, as any preliminary study, presents some limitations.

First, the exoskeleton was working in opened-loop control. This means that it was commanded by predefined instructions during the trials. However, as the final objective of the research is to develop a $\mathrm{BCI}$ taking into account the lateralization that works in closed-loop control, the findings should be tested in the future in this scenario.

Another concern is about the homogeneity of the database. All the participants were in their twenties and presented right-dominance for both hands and feet. This allows for a robust database, since they shared some common features, but further research should be done to generalize the findings to other population groups.

Another limitation lies in the fact that performing maintained gait imagery while wearing an exoskeleton can be difficult. Participants can suffer from fatigue, and it is more difficult to focus on the mental tasks, which usually makes for lower BCI accuracies [16].

\subsection{Algorithm Influence}

The purpose of this study was to gain a better understanding of hemisphere specialization during MI of the gait when using a BCI to control a lower-limb exoskeleton. It was studied for different subjects and across different sessions. Three processing algorithms were compared for the BCI-FBCSP, ST and Welch's PSD—and the highest results were obtained with FBCSP. This is consistent with previous investigations, which appointed FBCSP as a more robust method [40] The accuracy for full-static trials was $66.1 \pm 6.6 \%$ and for full-motion trials, $61.7 \pm 6.2 \%$.

\subsection{BCI Performance Comparison}

Only a few works in the literature have designed and evaluated a BCI based on maintained MI of the gait for controlling an exoskeleton without any other control paradigm based on external stimuli such as visual stimuli [7]. This pattern of results is consistent with the previous literature [41] and our previous research [16,42].

Whereas our previous research of a BCI based on MI of gait to control a treadmill [35] did not show differences between static and trials in motion, the present study has shown some variation. A possible explanation may be the higher complexity of walking with crutches assisted by an overground exoskeleton in comparison with walking on a treadmill.

\subsection{Subject Influence}

The results of this research provide supporting evidence that the performance of the $\mathrm{BCI}$ has significant differences among subjects [43]. Therefore, laterality of each subject was studied independently.

\subsection{Hemisphere Dominance}

Foot brain area is located in the interhemispheric vertex, so brain representations of each limb are very close [25]. Contrary to investigations of foot dorsiflexion MI [25], the MI of the gait was studied without considering each foot separately. Therefore, the brain lateralization of each limb was not studied, but the whole gait imagination process during two different scenarios was instead studied: full-static and full-motion.

In order to study the brain asymmetry, three electrode configurations were compared: electrodes covering both hemispheres, covering the non-dominant hemisphere (right hemisphere) and covering the dominant hemisphere (left hemisphere). There were no statistically significant differences among them in terms of performance. However, all subjects except S5 obtained the highest BCI average accuracy with information from both hemispheres when performing purely MI (full-static) and MI combined with robot-assisted gait (full-motion). S5 showed the highest results when using information from dominant hemisphere for trials in full-static MI and with information from non-dominant hemisphere 
for trials in full-motion MI with the exoskeleton assistance. These variations in brain asymmetry among subjects are consistent with the findings of Nielsen et al. [44].

The results from this research showed that the performance with electrodes on the dominant side (left) was slightly superior to the non-dominant side (right) for the majority of subjects. This differs from the findings of Crémers et al. [27] and Jahn et al. [28], which observed right hemisphere specialization during MI of the gait, especially in frontal and parietal areas. However, Sabate et al. [45] studied MI and motor execution of both hands in patients with unilateral brain damage, and they found that patients with left-side stroke decreased the velocity of movements, suggesting the left hemisphere plays a crucial role in motor planning. An fMRI investigation of gait MI identified dissimilar lateralization for brain regions involved in motor planning, as the posterior parietal cortex showed lateralization on the dominant hemisphere (left), and the activity of pre-supplementary motor was only found on non-dominant side (right) [46]. Although differences among electrode configurations were not statistically relevant, the usage of electrodes covering only the dominant hemisphere could reduce the preparation time. The reduction of the number of electrodes as well as the employment of dry or semi-dry electrodes would considerably reduce the preparation complexity and promote the usage of BCI in real-life scenarios $[47,48]$.

\subsection{Evolution of Performance with Practice}

Finally, the evolution of performance with practice was assessed for the different electrode configurations. In general, the evolution was positive, and the BCI accuracy of the last session was higher than the first one. These results are consistent with previous research [49]. However, this positive trend was more significant in full-static trials. In fact, for some subjects and electrode configurations, the evolution of performance of full-motion trials was negative. This difference can be attributable to the difficulty of performing mental tasks during robotic-assisted gait. In addition, the evolution of performance with practice was not statistically different among each electrode configuration.

\section{Conclusions}

The current research studied the brain lateralization in terms of performance of a BCI based on MI of the gait for controlling a lower-limb exoskeleton. This analysis was carried out for five different subjects who participated in five sessions. Significant differences were found among participants, so they were studied independently. For the majority of participants, the performance improved with training. Regarding brain lateralization, three different approaches were compared: EEG recording of both brain hemispheres, the left hemisphere and the right hemisphere. Results from each EEG setup were not statistically different, but the BCI with information from both hemispheres showed the highest accuracy. Collectively, our results showed that BCI accuracy was marginally higher with EEG data from the dominant hemisphere than the non-dominant hemisphere, and since all participants were right-footed, the dominant side was the left hemisphere. Future research might extend this research with more participants to validate the conclusions drawn in this study. If so, the BCI could use only information from half of the electrodes, reducing the setup preparation time. In addition, considering that the final user of this kind of BCI are people with spinal cord injury, future research should replicate this study with them, trying to identify whether there are similarities or differences in comparison to able-bodied subjects.

Supplementary Materials: The following are available online at https:/ / www.mdpi.com/article/10 .3390/sym13091746/s1, Figure S1: Boxplots representing the distribution of trial accuracies grouped by method and subject.

Author Contributions: Conceptualization, L.F. and M.O.; methodology, L.F., V.Q., M.O. and J.A.F.; software, L.F. and V.Q.; validation, M.O. and E.I.; formal analysis, L.F. and V.Q.; investigation, L.F. and V.Q.; resources, M.O., E.I., J.A.F. and J.M.A.; data curation, M.O.; writing-original draft preparation, 
L.F.; writing-review and editing, L.F., M.O. and J.M.A.; visualization, L.F.; supervision, M.O., E.I. and J.M.A.; project administration, J.M.A.; funding acquisition, J.M.A., J.A.F. and M.O. All authors have read and agreed to the published version of the manuscript.

Funding: This research was funded by the Spanish Ministry of Science and Innovation, the Spanish State Agency of Research and the European Union through the European Regional Development Fund in the framework of the project Walk-Controlling Lower-Limb Exoskeletons by Means of Brain-Machine Interfaces to Assist People with Walking Disabilities (RTI2018-096677-B-I00), and by the Consellería de Innovación, Universidades, Ciencia y Sociedad Digital (Generalitat Valenciana), and the European Social Fund in the framework of the project Desarrollo de Nuevas Interfaces Cerebro-Máquina Para la Rehabilitación de Miembro Inferior (GV /2019/009).

Institutional Review Board Statement: The study was conducted according to the guidelines of the Declaration of Helsinki, and approved by the Institutional Review Board of Miguel Hernandez University of Elche (DIS.JAP.03.18 and 22 January 2019).

Informed Consent Statement: Informed consent was obtained from all subjects involved in the study.

Data Availability Statement: Data not available to external researches due to restrictions of the ethical committee.

Conflicts of Interest: The authors declare no conflict of interest. The funders had no role in the design of the study; in the collection, analyses, or interpretation of data; in the writing of the manuscript; or in the decision to publish the results.

\section{References}

1. Mulder, T. Motor imagery and action observation: Cognitive tools for rehabilitation. J. Neural Transm. (Vienna Austria 1996) 2007, 114, 1265-1278. [CrossRef]

2. Lee, K.; Liu, D.; Perroud, L.; Chavarriaga, R.; Millán, J.d.R. A brain-controlled exoskeleton with cascaded event-related desynchronization classifiers. Robot. Auton. Syst. 2017, 90, 15-23. [CrossRef]

3. Pfurtscheller, G.; Neuper, C.; Andrew, C.; Edlinger, G. Foot and hand area mu rhythms. Int. J. Psychophysiol. 1997, 26, 121-135. [CrossRef]

4. Donati, A.R.; Shokur, S.; Morya, E.; Campos, D.S.; Moioli, R.C.; Gitti, C.M.; Augusto, P.B.; Tripodi, S.; Pires, C.G.; Pereira, G.A.; et al. Long-Term Training with a Brain-Machine Interface-Based Gait Protocol Induces Partial Neurological Recovery in Paraplegic Patients. Sci. Rep. 2016, 6, 30383. [CrossRef]

5. He, Y.; Eguren, D.; Azorín, J.M.; Grossman, R.G.; Luu, T.P.; Contreras-Vidal, J.L. Brain-machine interfaces for controlling lower-limb powered robotic systems. J. Neural Eng. 2018, 15. [CrossRef]

6. Soleimani Amiri, M.; Ramli, R.; Ibrahim, M.F.; Abd Wahab, D.; Aliman, N. Adaptive Particle Swarm Optimization of PID Gain Tuning for Lower-Limb Human Exoskeleton in Virtual Environment. Mathematics 2020, 8, 2040. [CrossRef]

7. Kwak, N.S.; Müller, K.R.; Lee, S.W. A convolutional neural network for steady state visual evoked potential classification under ambulatory environment. PLoS ONE 2017, 12, e0172578. [CrossRef]

8. Yu, Z.; Li, L.; Song, J.; Lv, H. The Study of Visual-Auditory Interactions on Lower Limb Motor Imagery. Front. Neurosci. 2018, 12, 509. [CrossRef] [PubMed]

9. Zhang, Y.; Prasad, S.; Kilicarslan, A.; Contreras-Vidal, J.L. Multiple kernel based region importance learning for neural classification of gait states from EEG signals. Front. Neurosci. 2017, 11, 170. [CrossRef] [PubMed]

10. Liu, D.; Chen, W.; Pei, Z.; Wang, J. A brain-controlled lower-limb exoskeleton for human gait training. Rev. Sci. Instr. 2017, 88, 104302. [CrossRef] [PubMed]

11. Kilicarslan, A.; Grossman, R.G.; Contreras-Vidal, J.L. A robust adaptive denoising framework for real-time artifact removal in scalp EEG measurements. J. Neural Eng. 2016, 13, 026013. [CrossRef]

12. Pfurtscheller, G.; Neuper, C.; Flotzinger, D.; Pregenzer, M. EEG-based discrimination between imagination of right and left hand movement. Electroencephalogr. Clin. Neurophysiol. 1997, 103, 642-651. [CrossRef]

13. Pfurtscheller, G.; Lopes Da Silva, F.H. Event-related EEG/MEG synchronization and desynchronization: Basic principles. Clin. Neurophysiol. 1999, 110, 1842-1857. [CrossRef]

14. Seeland, A.; Manca, L.; Kirchner, F.; Kirchner, E.A. Spatio-temporal comparison between ERD/ERS and MRCP-based movement prediction. In Proceedings of the BIOSIGNALS 20158th International Conference on Bio-Inspired Systems and Signal Processing, Proceedings, Part of 8th International Joint Conference on Biomedical Engineering Systems and Technologies, BIOSTEC 2015, Lisbon, Portugal, 12-15 January 2015; pp. 219-226. [CrossRef]

15. Choi, J.; Kim, K.T.; Jeong, J.H.; Kim, L.; Lee, S.J.; Kim, H. Developing a Motor Imagery-Based Real-Time Asynchronous Hybrid BCI Controller for a Lower-Limb Exoskeleton. Sensors 2020, 20, 7309. [CrossRef] [PubMed]

16. Ferrero, L.; Quiles, V.; Ortiz, M.; Iáñez, E.; Azorín, J.M. A BMI Based on Motor Imagery and Attention for Commanding a Lower-Limb Robotic Exoskeleton: A Case Study. Appl. Sci. 2021, 11, 4106. [CrossRef] 
17. Gharabaghi, A. What Turns Assistive into Restorative Brain-Machine Interfaces? Front. Neurosci. 2016, 10, 456. [CrossRef] [PubMed]

18. Tomasi, D.; Volkow, N.D. Laterality patterns of brain functional connectivity: Gender effects. Cereb. Cortex (New York NY 1991) 2012, 22, 1455-1462. [CrossRef]

19. Ocklenburg, S.; Hirnstein, M.; Beste, C.; Güntürkün, O. Lateralization and cognitive systems. Front. Psychol. 2014, 5, 1143. [CrossRef]

20. Sokołowska, B. A novel virtual reality approach for functional lateralization in healthy adults. Brain Res. 2021, $1766,147537$. [CrossRef]

21. Parsons, L.M.; Gabrieli, J.D.; Phelps, E.A.; Gazzaniga, M.S. Cerebrally lateralized mental representations of hand shape and movement. J. Neurosci. 1998, 18, 6539-6548. [CrossRef]

22. Zapała, D.; Zabielska-Mendyk, E.; Augustynowicz, P.; Cudo, A.; Jaśkiewicz, M.; Szewczyk, M.; Kopiś, N.; Francuz, P. The effects of handedness on sensorimotor rhythm desynchronization and motor-imagery BCI control. Sci. Rep. 2020, 10, 2087. [CrossRef]

23. Shieh, C.P.; Yang, S.H.; Liu, Y.S.; Kuo, Y.T.; Lo, Y.C.; Kuo, C.H.; Chen, Y.Y. Simultaneously Spatiospectral Pattern Learning and Contaminated Trial Pruning for Electroencephalography-Based Brain Computer Interface. Symmetry 2020, 12, 1387. [CrossRef]

24. Pfurtscheller, G.; Brunner, C.; Schlo, A.; Lopes, F.H. Mu rhythm (de) synchronization and EEG single-trial classification of different motor imagery tasks. NeuroImage 2006, 31, 153-159. [CrossRef]

25. Tariq, M.; Trivailo, P.M.; Simic, M. Mu-Beta event-related (de)synchronization and EEG classification of left-right foot dorsiflexion kinaesthetic motor imagery for BCI. PLoS ONE 2020, 15, e0230184. [CrossRef]

26. Tariq, M.; Uhlenberg, L.; Trivailo, P.; Munir, K.S.; Simic, M. Mu-beta rhythm ERD/ERS quantification for foot motor execution and imagery tasks in BCI applications. In Proceedings of the 2017 8th IEEE International Conference on Cognitive Infocommunications (CogInfoCom), Debrecen, Hungary, 11-14 September 2017; pp. 91-96. [CrossRef]

27. Crémers, J.; Dessoullières, A.; Garraux, G. Hemispheric specialization during mental imagery of brisk walking. Hum. Brain Mapp. 2012, 33, 873-882. [CrossRef]

28. Jahn, K.; Deutschländer, A.; Stephan, T.; Strupp, M.; Wiesmann, M.; Brandt, T. Brain activation patterns during imagined stance and locomotion in functional magnetic resonance imaging. NeuroImage 2004, 22, 1722-1731. [CrossRef]

29. Ang, K.K.; Chin, Z.Y.; Zhang, H.; Guan, C. Filter Bank Common Spatial Pattern (FBCSP) in brain-computer interface. In Proceedings of the International Joint Conference on Neural Networks, Hong Kong, China, 1-8 June 2008; pp. $2390-2397$. [CrossRef]

30. Ortiz, M.; Rodríguez-Ugarte, M.; Iáñez, E.; Azorín, J.M. Application of the Stockwell Transform to Electroencephalographic Signal Analysis during Gait Cycle. Front. Neurosci. 2017, 11, 660. [CrossRef] [PubMed]

31. Solomon, O.M.J. PSD Computations Using Welch's Method. [Power Spectral Density (PSD)]; Technical Report; Sandia National Labs.: Albuquerque, NM, USA, 1991. [CrossRef]

32. Ramoser, H.; Müller-Gerking, J.; Pfurtscheller, G. Optimal Spatial Filtering of Single Trial EEG During Imagined Hand Movement. IEEE Trans. Rehabil. Eng. A Publ. IEEE Eng. Med. Biol. Soc. 2001, 8, 441-446. [CrossRef] [PubMed]

33. McFarland, D.J.; McCane, L.M.; David, S.V.; Wolpaw, J.R. Spatial filter selection for EEG-based communication. Electroencephalogr. Clin. Neurophysiol. 1997, 103, 386-394. [CrossRef]

34. Izenman, A. Linear Discriminant Analysis. In Modern Multivariate Statistical Techniques; Springer Texts in Statistics; Springer: New York, NY, USA, 2013.

35. Ferrero, L.; Quiles, V.; Ortiz, M.; Iáñez, E.; Azorín, J.M. BCI Based on Lower-Limb Motor Imagery and a State Machine for Walking on a Treadmill. In Proceedings of the International IEEE EMBS Conference on Neural Engineering, Virtual, Italy, 4-6 May 2021.

36. Ferrero, L.; Ortiz, M.; Quiles, V.; Iáñez, E.; Azorín, J.M. Improving Motor Imagery of Gait on a Brain-Computer Interface by Means of Virtual Reality: A Case of Study. IEEE Access 2021, 9, 49121-49130. [CrossRef]

37. Tariq, M.; Trivailo, P.M.; Simic, M. Classification of left and right foot kinaesthetic motor imagery using common spatial pattern. Biomed. Phys. Eng. Express 2019, 6, 15008. [CrossRef] [PubMed]

38. Zhang, C.; Eskandarian, A. A Computationally Efficient Multiclass Time-Frequency Common Spatial Pattern Analysis on EEG Motor Imagery. In Proceedings of the 2020 42nd Annual International Conference of the IEEE Engineering in Medicine \& Biology Society (EMBC), Montreal, QC, Canada, 20-24 July 2020.

39. Leeuwis, N.; Paas, A.; Alimardani, M. Vividness of Visual Imagery and Personality Impact Motor-Imagery Brain Computer Interfaces. Front. Hum. Neurosci. 2021, 15, 634748. [CrossRef]

40. Lotte, F.; Bougrain, L.; Cichocki, A.; Clerc, M.; Congedo, M.; Rakotomamonjy, A.; Yger, F. A review of classification algorithms for EEG-based brain-Computer interfaces: A 10 year update. J. Neural Eng. 2018, 15, 031005. [CrossRef]

41. Gordleeva, S.Y.; Lukoyanov, M.V.; Mineev, S.A.; Khoruzhko, M.A.; Mironov, V.I.; Kaplan, A.Y.; Kazantsev, V.B. Exoskeleton control system based on motor-imaginary brain-computer interface. Sovrem. Tehnol. V Med. 2017, 9, 31-36. [CrossRef]

42. Ortiz, M.; Ferrero, L.; Iáñez, E.; Azorín, J.M.; Contreras-Vidal, J.L. Sensory Integration in Human Movement: A New BrainMachine Interface Based on Gamma Band and Attention Level for Controlling a Lower-Limb Exoskeleton. Front. Bioeng. Biotechnol. 2020, 8, 735. [CrossRef] 
43. Zhang, T.; Liu, T.; Li, F.; Li, M.; Liu, D.; Zhang, R.; He, H.; Li, P.; Gong, J.; Luo, C.; et al. Structural and functional correlates of motor imagery BCI performance: Insights from the patterns of fronto-parietal attention network. NeuroImage 2016, 134, 475-485. [CrossRef] [PubMed]

44. Nielsen, J.A.; Zielinski, B.A.; Ferguson, M.A.; Lainhart, J.E.; Anderson, J.S. An Evaluation of the Left-Brain vs. Right-Brain Hypothesis with Resting State Functional Connectivity Magnetic Resonance Imaging. PLoS ONE 2013, 8, e71275.

45. Sabaté, M.; Gonzalez, B.; Díaz, M. Brain lateralization of motor imagery: Motor planning asymmetry as a cause of movement lateralization. Neuropsychologia 2004, 42, 1041-1049. [CrossRef]

46. Labriffe, M.; Annweiler, C.; Amirova, L.E.; Gauquelin-Koch, G.; Ter Minassian, A.; Leiber, L.M.; Beauchet, O.; Custaud, M.A.; Dinomais, M. Brain Activity during Mental Imagery of Gait Versus Gait-Like Plantar Stimulation: A Novel Combined Functional MRI Paradigm to Better Understand Cerebral Gait Control. Front. Hum. Neurosci. 2017, 11, 106. [CrossRef]

47. Li, G.L.; Wu, J.T.; Xia, Y.H.; He, Q.G.; Jin, H.G. Review of semi-dry electrodes for EEG recording. J. Neural Eng. 2020, $17,51004$. [CrossRef]

48. Li, G.; Wang, S.; Li, M.; Duan, Y.Y. Towards real-life EEG applications: Novel superporous hydrogel-based semi-dry EEG electrodes enabling automatically 'charge-discharge' electrolyte. J. Neural Eng. 2021, 18, 046016. [CrossRef] [PubMed]

49. Zich, C.; De Vos, M.; Kranczioch, C.; Debener, S. Wireless EEG with individualized channel layout enables efficient motor imagery training. Clin. Neurophysiol. 2015, 126, 698-710. [CrossRef] [PubMed] 